\title{
Original Research Article \\ Cancer scenario at a tertiary care teaching hospital in rural Maharashtra
}

\author{
Jaison J. ${ }^{1}$, Zope R.D. ${ }^{2}$, Bhide S.P. ${ }^{3}$, Joshi S. R. ${ }^{4}$ \\ ${ }^{1}$ Dr. Janice Jaison, Assistant Professor, ${ }^{2}$ Dr. Rajendra D. Zope, Associate Professor, ${ }^{3}$ Dr. Smita P. Bhide, Professor, \\ ${ }^{4}$ Dr. S. R. Joshi, Professor and HOD, all authors are affiliated with Department of Pathology, MIMER Medical College, \\ Talegaon Dabhade Pune, India.
}

Corresponding Author: Dr. Rajendra D Zope, Associate Professor, Department of Pathology, MIMER Medical College, Talegaon Dabhade, Pune, India. E-mail: zope_rajendra@yahoo.com

\begin{abstract}
Introduction: Cancer is a major public health problem, which is increasing at a rapid rate. Study of the magnitude of the problem and the type of cancers prevalent in our local setting will help to plan and assess control measures. This study was carried out at a tertiary care teaching hospital in rural Maharashtra, with the study population mainly from rural areas and most of the patients belonging to low socioeconomic status. Methods: This is a retrospective observational study analyzing data over a period of 3 years. Results: Malignancies constituted $2 \%$ of the total histopathological specimens received in the Department of Pathology. The peak incidence of malignancies was noted in the age group 41-70 years. Female patients outnumbered male patients marginally. The leading 5 malignancies in our set up were malignancies of Oral Cavity, followed by Gastrointestinal tract, Female genital tract, Breast and Male genital tract malignancies. In males, Oral cavity malignancies were the commonest and the main site involved was the buccal mucosa, while in females, malignancies of Female genital tract were the leading malignancy with cervical cancer being the commonest. Conclusion: Various activities for awareness, prevention, early detection and treatment are being carried out in our institute. The importance of rural cancer registries is also emphasized.
\end{abstract}

Key words: Malignant neoplasms, Rural area, Awareness, Early detection

\section{Introduction}

Cancer is being increasingly recognized as a major contributor to health concerns globally [1]. There are about 2.5 million cancer cases in India. Nearly 8, 00,000 new cases are diagnosed and 5, 50,000 deaths occur annually due to cancer [2].

The top five cancers in men in India are cancers of lip and oral cavity, lung, stomach, colorectum and pharynx while in women cancers of breast, cervix, colorectum, ovary, lip and oral cavity are the commonest.

These cancers can be screened for and or detected early and treated at an early stage. Environmental factors and lifestyle changes are said to play a key role in determining the geographical patterns of cancer [3].

The alarming increase in morbidity and mortality due to cancer is a major concern to the society.

Manuscript received: $20^{\text {th }}$ April 2019

Reviewed: $30^{\text {th }}$ April 2019

Author Corrected: $7^{\text {th }}$ May 2019

Accepted for Publication: 12 ${ }^{\text {th }}$ May 2019

\section{Aims and Objectives}

1. To evaluate the common malignancy amongst all system malignancies at a tertiary care medical college hospital.

2. To plan preventive measures and awareness programs related to the common malignancies in our set up.

\section{Materials and Methods}

Setting and Type of Study: This study was carried out at a Tertiary care Teaching Hospital in Rural Maharashtra. It is a Retrospective Observational Study

Sampling Method and Sample Collection: The data was collected from all histopathological specimens received in the Department of Pathology of a tertiary care medical college hospital over a period of three years.

Inclusion Criteria: All cases of malignancies reported among the histopathological specimens received in the Department of Pathology were included in the study. 
Exclusion criteria: Nonmalignant cases among the histopathological specimens received in the Department of Pathology were excluded in the study.

The malignant cases included in the study were studied with respect to age, gender, site of the lesion and

\section{Original Research Article}

histopathological diagnosis. The data collected was analyzed according to the various above mentioned parameters.

The findings were summarized in appropriate tables using percentages.

\section{Results}

A total of 10,627 histopathological specimens were received in the Department of Pathology over a period of 3 years, of which 213(2\%) malignant tumors were reported.

Table-1: Age wise Distribution of Cases.

\begin{tabular}{|c|c|}
\hline Age Distribution & Number of Cases \\
\hline $0-20$ & 1 \\
\hline $21-30$ & 9 \\
\hline $31-40$ & 19 \\
\hline $41-50$ & 53 \\
\hline $51-60$ & 45 \\
\hline $61-70$ & 61 \\
\hline $71-80$ & 22 \\
\hline $81-90$ & 1 \\
\hline $91-100$ & 2 \\
\hline
\end{tabular}

There were 61 cases $(28.63 \%)$ of malignancies were in the age group of $61-70$ years, followed by 53 cases $(24.88 \%)$ in the age group $41-50$ years and 45 cases $(21.12 \%)$ in the age group 51-60 years. (Table1). A peak incidence of malignancies was noted in the age group $41-70$ years $(74.63 \%)$ (Table 1$)$

Table-2: Sex wise Distribution of Cases.

\begin{tabular}{|c|c|c|}
\hline Sex & Number of Cases & Percentage of cases \\
\hline Females & 107 & 50.23 \\
\hline Males & 106 & 49.77 \\
\hline
\end{tabular}

No significant differences were noted in the sex distribution among malignant cases.50.23\% of total malignancies occurred in female patients while $49.77 \%$ malignancies were found in male patients (Table 2)

Table-3: System wise distribution of cases.

\begin{tabular}{|c|c|}
\hline System & Number of cases \\
\hline Oral Cavity & 55 \\
\hline GIT & 37 \\
\hline FGS & 33 \\
\hline Breast & 29 \\
\hline MGS & 14 \\
\hline RS & 13 \\
\hline Skin & 12 \\
\hline Lymph Node \&Spleen & 5 \\
\hline Endocrine & 4 \\
\hline Urinary System & 3 \\
\hline Soft tissue & 3 \\
\hline Salivary gland & 2 \\
\hline Eye & 2 \\
\hline Liver & 1 \\
\hline Total & $\mathbf{2 1 3}$ \\
\hline
\end{tabular}




\section{Original Research Article}

The 5 leading malignancies in our study constituted malignancies of Oral Cavity, followed by Gastrointestinal tract, Female genital tract, Breast and Male genital tract malignancies. Malignancies of the oral cavity was the commonest constituting $25.82 \%$, followed by gastrointestinal tract malignancies constituting $17.37 \%$, female genital tract malignancies $15.49 \%$, Breast $13.61 \%$ and Male genital tract malignancies $6.57 \%$ (Table 3)

In males, the commonest malignancy seen was malignancies of the Oral cavity $(34.90 \%)$, followed by malignancies of Gastrointestinal tract (19.81\%), Male genital system (13.20\%), Respiratory system (10.37\%) and malignancies of Skin $(6.60 \%)$

In females, the top 5 malignancies constituted malignancies of Female genital tract $(28.03 \%)$, followed by Breast (26.16\%), Oral cavity (16.82\%), Gastrointestinal tract (14.95\%) and Skin (4.67\%).

Oral malignancies constituted $25.82 \%$ of total malignancies. The youngest age for oral malignancy was 33 years while the oldest patient was a 90 year old male patient.Most cases (29.09\%) were observed in the age group 61-70 years. Oral malignancies were more common in male constituting $67.27 \%$. The most common site for oral malignancies was the buccal mucosa ( 25 cases), followed by lip (11 cases), tongue ( 9 cases), cheek ( 6 cases), palate ( 3 cases) and a single case involving the tonsil. The most common histological type of oral malignancy was Squamous cell carcinoma, followed by 2 cases of Verrucous carcinoma and 1 case of low grade malignancy (Epitheloid Haemangioma)

Gastrointestinal malignancies constituted (17.37\%) of the total malignancies. GI malignancies were more common in males $(56.75 \%)$ as compared to females. The youngest patient with GI malignancy was a 21 year old female patient while the oldest patient was a 78 year old male patient. Colonic carcinomas were the commonest $(37.83 \%)$ followed by gastric adenocarcinomas (27.02\%), esophageal malignancies (16.21\%) and small bowel malignancy $(8.10 \%)$.

In the female genital tract, there were 20 cases of cervical cancer. The youngest patient was 20 years old while the oldest was 70 years old female. Majority of the patients with cervical cancer were in the $4^{\text {th }}$ to $6^{\text {th }}$ decade $(85 \%)$. The histological types of cervical cancers studied were, 8 cases each of Keratinizing Squamous cell carcinoma and Non keratinizing Squamous cell carcinoma, and a single case each of Glassy cell carcinoma, Mucinous Adenocarcinoma, Undifferentiated Carcinoma and Verrucous carcinoma.

There were a total of 4 cases of Endometrial carcinoma, 2 cases of Endometroid adenocarcinoma while a single case each of Keratinizing Well differentiated Squamous cell carcinoma and Serous Carcinoma. There were 7 cases of ovarian tumors, Surface epithelial tumors were the most common $(71.42 \%)$, of which there were 2 cases of Mucinous cystadenocarcinoma, one case each of Serous cystadenocarcinoma and borderline serous tumor and borderline seromucinous tumor. There was a single case each of Adult Granulosa cell tumor and a single case of Primary Malignant Melanoma of the ovary. There were 2 cases of Squamous cell Carcinoma, one involving vulva and the other involving the vaginal vault.

There were 29 cases of Breast malignancies, of which 28 were in female patients and there was a single case of breast malignancy in a male patient. Out of 29 cases, Infiltrating duct carcinoma (NST) was the commonest (75.86\%), followed by Mucinous carcinoma (10.34\%), Lobular carcinoma (6.89\%) and Medullary carcinoma and Borderline Phylloides tumor $(3.44 \%$ each $)$

In Male Reproductive System, there was a total of 14 malignancies, 8 Prostatic Adenocarcinoma, 5 Penile carcinomas, out of which 4 were Squamous Cell carcinoma and one of Verrucous Carcinoma and a single case of Immature Teratoma with YST arising from the testis.

Out of 13 cases of Respiratory tract, 10 cases were cancers involving larynx, followed by 2 cases arising from nasopharynx and 1 case of primary lung cancer. All the laryngeal cancers were Squamous cell carcinoma, while the 2 cases of nasopharyngeal tumors were Nasopharyngeal carcinoma and there was a single case of Small cell carcinoma of Lung.

Out of the 12 cutaneous tumors, there were 4 cases of Basal cell carcinoma, 3 of squamous cell carcinoma, 2 of Malignant melanoma, 1 of verrucous carcinoma and 2 cases of metastatic tumor deposits, one from adenocarcinoma and one from squamous cell carcinoma. 


\section{Original Research Article}

In the lymphoreticular system, there were 3 cases of NHL and one case of HD and a single case of Splenic Lymphoma Endocrine tumors constituted $1.87 \%$ of the total malignancies. There were 3 cases of Thyroid malignancies, 2 of Follicular carcinoma and 1 of Papillary carcinoma. The single case of Pancreatic tumor in a 30 year old female was Papillary and Solid Epithelial Neoplasia.

3 cases of Bladder tumors were studied. All the 3 cases were High grade Invasive Urothelial Carcinoma. There were 3 cases of Soft tissue malignancies- one case of Malignant Spindle cell tumor - Pleomorphic sarcoma, one case of Malignant small round cell tumor and one case of a high grade tumor, for both of which IHC was advised.

There were 2 cases of Salivary gland Malignancies- one of Salivary duct carcinoma arising in the submandibular gland and one case of invasive carcinoma ex pleomorphic adenoma arising from the parotid gland.

There were 2 cases of Eye tumors- one was diagnosed as an malignant epithelial tumor- Squamous cell Carcinoma/ Malignant melanoma and one case of Pigmented Squamous cell Carcinoma.A single case of Hepatocellular carcinoma was diagnosed on Liver biopsy.

\section{Discussion}

Cancer is a major public health problem, which is increasing at a rapid rate and it has become a leading cause of death worldwide. Study of the magnitude and pattern of cancer helps in determining clues to the cause of cancer and in forming a basis, to plan and assess control measures. The present study was carried out at a tertiary care hospital with the study population mainly from rural areas and most of the patients were from low socioeconomic group.

The incidence of malignancy in our study was $2 \%$. This is comparable with the studies carried out by Pawar S. et al (3.13\%) and Mankar R et al (3.83\%). Similar study was carried out by Malhotra $\mathrm{V}$ et al, where a slightly higher incidence of malignancy $(8.36 \%)$ was noted $[4$, $5,6]$.

Maximum number of patients were found in 41-70 years age group $(74.63 \%)$. Similar findings were also found in other studies [3- 6]. A female preponderance was noted in the present study and is comparable with the other studies [3-6]. In the present study, malignancies of the oral cavity were commonest in males followed by Gastrointestinal tract malignancy and malignancies of the male genital system, while in females, malignancies of the female genital tract were commonest followed by $\mathrm{Ca}$ breast and malignancies of oral cavity.

In studies carried out by Pawar S et al, malignancies of the female genital tract were commonest followed by breast malignancies and oral cancers [4]. According to Malhotra $\mathrm{V}$ et al in males, malignancy of hypopharynx and larynx are the commonest followed by prostate cancer and malignancy of esophagus, while in female breast cancers are the commonest followed by cervical cancers and carcinoma of esophagus [6]. Oral cavity malignancies were commonest followed by lung and
GIT malignancy in males whereas cervical cancers were commonest followed by breast cancers in females, according to studies carried out by Deshpande et al and Mankar et al [3,5].

Lack of awareness among the people, self-neglect, late presentation, more number of quacks who do not have any knowledge of cancer and its management and who play a major role in health management at grass root levels, increase use of tobacco, alcohol etc, poverty and limited resources all add to the increased burden of cancer in rural areas [3]. In most of the studies, including the present study there is a high prevalence of oral cavity malignancies, due to increased use of tobacco particularly among the rural population. Earlymarriage, low socioeconomic status of women, low educational level, age at first childbirth and parity among the local population are important risk factors for cervical cancer [3].

According to the National cancer registry program the leading sites of cancer is the oral cavity, lung, esophagus and stomach in men and cervix, breast and oral cavity in women. Educational, research, prevention and treatment programs aimed at fighting are extensively being explored and implemented. The government of India first developed its statement on cancer control in 1971 which was revised in 1984 with the aim to have primary prevention of tobacco related cancers, early detection of cancers of easily accessible sites, alignment of treatment facilities and providing pain and palliative care [2]. Some of the strategies that can be used for cancer prevention particularly in rural areas include increasing awareness about cancer by educating the public about common symptoms and signs of cancer and importance of early diagnosis and treatment. Educating health care workers at grass root level is very important [2]. 
Sensitization of school children and youth by conducting debates, discussions, street play competitions will help in the long run. Use of wellillustrated audio-visual educational materials are extremely useful for literate as well as illiterate population. Participation of Health administration, NGOs and all involved in health care with a common goal is essential [2].

Oral cancer is the leading cause of cancer among males in India. In rural areas the high prevalence of chewing betel quid and smoking is one of the major factors contributing to oral cancers. Many people in rural areas are still ignorant of the fact that these habits can cause cancer. Educating the common man about the harmful effects of these habits is essential. Also educating the health workers about clinical examination of oral cavity is the best and cheapest method for diagnosing premalignant and malignant lesions [2].

Cervical cancer is the most common cancer in Indian women. Cytologic screening is the most effective method for detecting and treatment of precancerous lesions. In rural areas, initial visual inspection with acetic acid (VIA) or Lugols iodine (VILI) by trained personal can be carried out and patients can be referred to higher Centre for Pap screening. Educating the female health workers about signs and symptoms of cervical cancer and knowledge about referral centres is essential to curb this menace [2].

Breast cancer is the second most common type of cancer in Indian women. Clinical breast examination by trained personnel in rural areas like female health workers is of utmost importance at remote places. A number of programs are being conducted by National Rural health mission like organizing training and education for the health workers regarding clinical breast examination [2].

In our institution various educational activities are carried out to promote awareness about cancer, particularly with respect to prevention and early detection. Cytological screening of leukoplakia lesions or biopsy depending upon the clinical relevance are being carried out. Counselling of patients is emphasized. Importance of follow up is also explained to the patients. For cervical pathologies, Pap smear examination is routinely performed. Self breast examination awareness is also promoted.

All these activities are carried out both in the Outpatient Department of our institute as well as in the Rural Health Centre and in the community in association with the various clinical departments and Community

\section{Original Research Article}

Medicine Department. The faculty and students are also encouraged to carry out projects pertaining to early cancer detection and prevention.

Relevance of population based cancer registries for planning and implementing cancer control programs is highly recommended. There are very few rural registries in India. Innovative methods for cancer registration in rural areas needs to be initiated [7].

\section{Conclusion}

Malignancies constituted $2 \%$ of the total histopathological specimens received in the Department of Pathology. The peak incidence of malignancies was noted in the age group 41-70 years. Female patients out numbered male patients marginally.

The leading 5 malignancies in our set up were malignancies of Oral Cavity followed by Gastrointestinal tract, Female genital tract, Breast and Male genital tract malignancies.

In males, Oral cavity malignancies were the commonestand the main site involved was the buccal mucosa, while in females, malignancies of Female genital tract were the leading malignancy with cervical cancer being the commonest. Most patients in our rural setup mainly presented late in the course of the disease. The reasons for the late presentation were identified with some studies especially studies done by S Das et al. Steps towards emphasis on awareness programs and early cancer detection have been undertaken in our institution, particularly with reference to the common malignancies in our area.

What this study adds to the existing knowledge: There is very few data available regarding cancer studies in the rural setup. This study contributes to the data regarding Cancer profile at a tertiary care teaching hospital in rural Maharashtra.

Similar studies would help to plan and implement cancer control programs targeting mainly the rural population.

Contribution by authors: Dr Janice Jaison and Dr Rajendra Zope contributed to the concept and design of the study, review of literature, compilation and interpretation of data, manuscript preparation and editing. Dr Smita P Bhide and Dr S.R. Joshi gave valuable inputs, guidance and final approval of the drafted article.

Findings: Nil; Conflict of Interest: None initiated Permission from IRB: Yes 


\section{References}

1. Gandhi AK, Kumar P, Bhandari M, et al. Burden of preventable cancers in India: Time to strike the cancer epidemic. J Egypt Natl Canc Inst. 2017 Mar;29(1):1118. doi: 10.1016/j.jnci.2016.08.002. Epub 2016 Aug 30.

2. Das S, Patro KC. Cancer care in the rural areas of India: a firsthand experience of a clinical oncologist and review of literatures. J Cancer Res Ther. 2010 Jul-Sep;6 (3): 299-303. doi: 10.4103/0973-1482.73369.

3. Deshpande JD, Singh KK, Phalke DB. Profile of Cancer Cases at a Tertiary Care Level Teaching Hospital in Rural Western Maharashtra, India. Natl J of Community Med 2012; 3(4):607- 11.

4.ShubhangiPawar, KanchanmalaGhorpade, Archana Khandelwar, Shilpa Gupta and Ashish Saraf. An overview of malignancy cases reported on histopathology over a span of 5 years (2011-2015) at Terna Medical College, Nerul, Navi Mumbai. J Res

\section{Original Research Article}

Med Dent Sci, 2017, 5(1):19-22. DOI: 10.5455/jrmds. 2017514. eISSN No. 2347-2367: pISSN No. 2347-2545

5. B.B.Sonawa, Reshma Dinesh Mankar, Study Of Spectrum Of Neoplastic Lesions In North West Maharashtra. International Journal Of Scientific Research And Education, [S.L.], V. 5, N. 09, Sep. 2017. Issn 2321-7545. Available At: <Https://Ijsae.In/Index. Php / Ijsae/Article/View/252>. Date Accessed: 24 May 2019.

6. V Malhotra, BS Shah, S Sabharwal. Pattern of Cancer in Dayanand Medical College and Hospital Ludhiana. Indian J Pathol Microbiol 2001;44(1): 27-30.

7. Kasturi Jayant, Bhagwan M Nene, Rajendra A Badwe, Nandkumar S Panse, Ranjit V Thorat, Feroz Y Khann. Rural cancer registry at Barshi Maharashtra and its impact on cancer control. Natl Med J India 2010; 23:274-7

\section{How to cite this article?}

Jaison J, Zope R.D, Bhide S.P, Joshi S. R. Cancer scenario at a tertiary care teaching hospital in rural Maharashtra. Trop J Path Micro 2019;5(5):269-274.doi:10.17511/jopm.2019.i05.03. 